\title{
The Predictive Role of Systemic Inflammation Response Index (SIRI) in the Prognosis of Stroke Patients
}

\author{
Yihui Zhang $\mathbb{D}^{1,2}$ \\ Zekun Xing ${ }^{3}$ \\ Kecheng Zhou ${ }^{1,2}$ \\ Songhe Jiang ${ }^{1,2}$
}

'Rehabilitation Medicine Center, The Second Affiliated Hospital and Yuying Children's Hospital, Wenzhou Medical University, Wenzhou, 325000, Zhejiang, People's Republic of China; ${ }^{2}$ Intelligent Rehabilitation Research Center, ChinaUSA Institute for Acupuncture and Rehabilitation, Wenzhou Medical University, Wenzhou, Zhejiang, People's Republic of China; ${ }^{3}$ Neurology Department, Wencheng People's Hospital, Wenzhou, Zhejiang, People's Republic of China
Correspondence: Songhe Jiang Rehabilitation Medicine Center, The Second Affiliated Hospital and Yuying Children's Hospital, No. 109, West College Road, Wenzhou, Zhejiang, People's Republic of China

Email jiangsonghe@wmu.edu.cn
Purpose: Stroke is a disease associated with high mortality. Many inflammatory indicators such as neutrophil-to-lymphocyte ratio (NLR), platelet-to-lymphocyte ratio (PLR), lymphocyte to monocyte ratio (LMR) and red blood cell distribution width (RDW) have been documented to predict stroke prognosis, their predictive power is limited. A novel inflammatory indicator called systemic inflammatory response index (SIRI) has been advocated to have an essential role in the prognostic assessment of cancer and infectious diseases. In this study, we attempted to assess the prognosis of stroke by SIRI. Moreover, we compared SIRI with other clinical parameters, including NLR, PLR, LMR and RDW.

Methods: This was a retrospective cohort study. We obtained data of 2450 stroke patients from the Multiparametric Intelligent Monitoring in Intensive Care III database. We used the Cox proportional hazards models to evaluate the relationship between SIRI and all-cause mortality and sepsis. Receiver operating curve (ROC) analysis was used to assess the predictive power of SIRI compared to NLR, PLR, LMR and RDW for the prognosis of stroke. We collected data of 180 patients from the First Affiliated Hospital of Wenzhou Medical University, which used the Pearson's correlation coefficient to assess the relationship between SIRI and the National Institute of Health stroke scale (NIHSS).

Results: After adjusting multiple covariates, we found that SIRI was associated with allcause mortality in stroke patients. Rising SIRI accompanied by rising mortality. Besides, ROC analysis showed that the area under the curve of SIRI was significantly greater than for NLR, PLR, LMR and RDW. Besides, Pearson's correlation test confirmed a significant positive correlation between SIRI and NIHSS.

Conclusion: Elevated SIRI was associated with higher risk of mortality and sepsis and higher stroke severity. Therefore, SIRI is a promising low-grade inflammatory factor for predicting stroke prognosis that outperformed NLR, PLR, LMR, and RDW in predictive power.

Keywords: systemic inflammation response index, stroke, NIHSS, mortality

\section{Introduction}

Stroke is an acute cerebrovascular disease caused by the sudden rupture of vessels in the brain or the inability of blood to flow to the brain due to the occlusion of vessels. ${ }^{1}$ Stroke is the second leading cause of death and a major contributor to disability in China. ${ }^{2}$ It is characterized by high morbidity, mortality, and disability and greatly burdens society and families. ${ }^{3}$ Ischemic stroke is highly predominant, being one of the most important causes of neurological morbidity and mortality. It is multifactorial in origin and influenced by 
multiple genetic and environmental risk factors. No definite mechanism has been found so far. ${ }^{4-6}$

Inflammation is a major factor in the pathology and outcome of acute ischemic stroke. ${ }^{7}$ Inflammation can induce secondary brain injury by exacerbating blood-brain barrier damage, microvascular failure, brain edema, oxidative stress, and directly inducing neuronal cell death. Accordingly, inflammation is currently considered a prime target for developing new stroke therapies. ${ }^{5}$ Inflammatory biomarkers are expected to help predict mortality and functional outcomes in stroke patients. ${ }^{8}$ As inflammatory indicators, the neutrophilto-lymphocyte ratio (NLR), platelet-to-lymphocyte ratio (PLR), lymphocyte to monocyte ratio (LMR) and red blood cell distribution width (RDW) have been reported to be predictive of stroke prognosis, but their formulations are relatively homogeneous and not comprehensive. ${ }^{9,10}$ It has been reported that the novel chronic low-grade inflammatory index systemic immunity-inflammation index (SIRI) has excellent predictive power in glioma, ${ }^{11}$ breast cancer ${ }^{12}$ and nasopharyngeal carcinoma; ${ }^{13}$ however, no studies have attempted to investigate its role in prediction in stroke prognosis.

SIRI is a more comprehensive marker for chronic lowgrade inflammation based on monocyte, neutrophil, and lymphocyte counts. ${ }^{14}$ Neutrophils are the forerunners to brain lesions after ischemic stroke and perform elaborate functions. After the onset of stroke, the neutrophil composition of peripheral blood increases shortly, and higher neutrophil counts have been associated with unfavorable stroke outcomes. ${ }^{15,16}$ Peripheral monocytes infiltrate the lesion site within 24 hours after ischemic stroke, and traditionally, monocytes are thought to play a deleterious role in ischemic stroke. ${ }^{17}$ The role of lymphocytes in stroke is complicated; in some settings, $\mathrm{T}$ cells still seem to aggravate neuronal damage late after the ischemic insult, while regulatory $\mathrm{B}$ cells were beneficial in mouse models of stroke. ${ }^{18,19}$

Interestingly, a recent study confirmed that SIRI was independently associated with ischemic stroke in patients with rheumatoid arthritis. ${ }^{20}$ Therefore, we aimed to assess the prognostic impact of SIRI on the mortality and severity of stroke patients.

\section{Materials and Methods}

\section{Data Acquisition}

Data for the study were obtained from the publicly available Multiparametric Intelligent Monitoring in Intensive
Care III (MIMIC-III) database, version 1.4. MIMIC-III includes identifiable health data for $>50,000$ critically ill patients enrolled to Beth Israel Deaconess Medical Center (Boston, MA, USA) from 2001 to $2012 .^{21}$ An Institutional Review Board endorsement was obtained from the Massachusetts Institute of Technology (Cambridge, MA, USA) and Beth Israel Deaconess Medical Center (Boston, MA, USA). All personal information was deleted to preserve the privacy of the patients.

The inclusion criteria were as follows:1. Patients who suffered from acute stroke; 2 . Hospitalization in an intensive care unit (ICU); 3. Hospitalization of at least 48 hours; 4. Age 16 years or older. Patients with more than $20 \%$ of missing data were excluded.

In addition, we collected data of 180 patients from the First Affiliated Hospital of Wenzhou Medical University between August 2019 and August 2021 to analyze the correlation between SIRI and NIHSS. This retrospective cohort study was approved by the Ethics Committee in Clinical Research of the First Affiliated Hospital of Wenzhou Medical University (registration number: KY2021-R104). The data were anonymous, and thus, the requirement for informed consent was discarded. This study was conducted in accordance with the Declaration of Helsinki.

\section{Study Variables and Outcomes}

The data included age, gender, race, vital signs, laboratory characteristics, comorbidities and metric scores. Vital signs included heart rate, systolic blood pressure (SBP), diastolic blood pressure (DBP), mean arterial pressure (MAP), respiratory rate and temperature. Complications included acute atrial fibrillation, coronary artery disease (CAD), chronic liver disease, chronic obstructive pulmonary disease (COPD), respiratory failure, peripheral vascular disease, sepsis, diabetes mellitus (DM) and hypertension. Besides, laboratory data included neutrophil count, monocyte count, lymphocyte count, white blood cell (WBC) count, hemoglobin, platelet count, red blood cell volume distribution width (RDW), glucose, serum creatinine and blood urea nitrogen (BUN) collected for the first 24 hours in the ICU. Metrics included the National Institute of Health stroke scale (NIHSS), Simplified Acute Physiology Score II (SAPS II) and Sequential Organ Failure Assessment (SOFA). The primary outcomes were the 90-day all-cause mortality, correlation analysis of SIRI and NIHSS and comparative analysis of SIRI with NLR, PLR and LMR. The 30-day, one-year and in- 
hospital all-cause mortality of stroke patients were secondary outcomes.

\section{Statistical Analyses}

The value of SIRI is continuous. Mean $\pm \mathrm{SD}$ was used to portray continuous variables, and categorical variables were presented as numbers and percentages. Multivariable cox regression and smooth curve fitting were used to analyze the independent effects of the SIRI levels and mortality in patients. The adjusted variables encompassed age, sex, ethnicity, systolic blood pressure, diastolic blood pressure, heart rate, glucose, anion gap, temperature, platelet counts, atrial fibrillation, liver disease, respiratory failure, serum creatinine, hemoglobin GCS, and SOFA. These confounders were selected since they have been documented to be associated with stroke prognosis, and had an estimated change in association with outcome of more than 10\%. Receiver Operating Characteristic (ROC) curve analysis was applied to compare the predictive power of SIRI with NLR, PLR, LMR and RDW for mortality in stroke patients. The Pearson correlation method was used to analyze the correlation between SIRI and NIHSS; a p-value less than 0.05 was considered statistically significant. All statistical analyses were performed with $\mathrm{R}$ software 4.0.0 (https://www.r-project.org/).

\section{Results}

\section{Demographic}

2450 patients from the MIMIC-III database were enrolled in this study. They were classified into three levels based on SIRI values. Tables 1 and $\underline{\mathrm{S} 1}$ demonstrate the features of these participants. As shown in Table 1, there were significant differences in heart rate, SBP, DBP, MAP, respiration rate, temperature, $\mathrm{WBC}$, platelet, glucose, BUN, anion gap, respiratory failure, pneumonia SOFA and SAPS II, but no differences in atrial fibrillation, CAD and liver disease. Moreover, the all-cause mortality (in-hospital, 30-days, 90-days and one year) of stroke patients increased with SIRI.

\section{Association Between SIRI and Clinical Outcomes}

The smooth curve fit in Figure 1 visually illustrates that allcause mortality at 30 days, 90 days and one year escalated significantly with increasing SIRI. The 90-day mortality was $21.9 \%(536 / 2450)$ (Table S1). Table 2 shows that a high SIRI was associated with the risk for 30-day, 90-day, one year and in-hospital all-cause mortality in stroke patients. For model 1, for 90-day mortality, the HR (95\% CI) for the second (1.6-3.8) and third (>3.8) tertiles were $1.21(0.99,1.48)$ and $1.80(1.49,2.18)$, respectively, compared to the first tertile $(<1.6)$. For Model 2, which was adjusted for age, sex and ethnicity, the HR (95\% CI) of 90day mortality for the second (1.6-3.8) and third (>3.8) tertiles were $1.19(0.97,1.46)$ and $1.75(1.45,2.11)$ respectively, compared to the first tertile $(<1.6)$.

A similar trend was found for model 3, after adjusting age, sex, ethnicity, systolic blood pressure, diastolic blood pressure, heart rate, glucose, anion gap, temperature, platelet counts, atrial fibrillation, liver disease, respiratory failure, serum creatinine, hemoglobin, GCS, and SOFA. The HR $(95 \% \mathrm{CI})$ for the second (1.6-3.8) and third (>3.8) tertiles were $1.31(1,04,1.65)$ and $1.42(1.13,1.78)$ respectively, compared to the reference $(<1.6)$. A similar trend was observed for the 30-day, one-year and in-hospital allcause mortality.

We observed another similar result to the mortality. As seen in Table 3, higher SIRI values were associated with a higher incidence of sepsis. For model 1, the OR $(95 \% \mathrm{CI})$ for the second (1.6-3.8) and third (>3.8) tertiles were $1.28(1.03,1.60)$ and $2.19(1.77,2.71)$, respectively, compared to the first tertile $(<1.6)$. For Model 2, which was adjusted for age, sex and ethnicity, the OR $(95 \% \mathrm{CI})$ for the second (1.6-3.8) and third (>3.8) tertiles were $1.32(1.06,1.65)$ and $2.26(1.82,2.81)$ respectively, compared to the first tertile $(<1.6)$. For model 3, after adjusting systolic blood pressure, diastolic blood pressure, system inflammatory response syndrome, serum creatinine, hemoglobin, white blood cell count, platelet count, red cell volume distribution width, atrial fibrillation, coronary artery disease, chronic kidney disease, respiratory failure, pneumonia, GCS and SOFA. The OR $(95 \% \mathrm{CI})$ for the second (1.6-3.8) and third $(>3.8)$ tertiles were $1.20(0.92,1.57)$ and $1.45(1.10$, 1.91) respectively, compared to the first tertile $(<1.6)$.

We performed a subgroup analysis and found that the results were stable (Table 4 ).

\section{ROC Curve Analysis for 90-Day Mortality}

ROC curves were plotted to assess the efficiency of SIRI and NLR, PLR, LMR and RDW in predicting mortality in stroke patients in Figure 2 and Table 5. We found that SIRI was more accurate than other biomarkers of inflammation including NLR, PLR, LMR and RDW (AUC 0.6216 vs 
Table I Baseline Characteristics of the Study Population

\begin{tabular}{|c|c|c|c|c|}
\hline \multirow[t]{2}{*}{ Characteristics } & \multicolumn{3}{|c|}{ SIRI } & \multirow[t]{2}{*}{$P$ value } \\
\hline & $<1.6$ & I.6-3.8 & $>3.8$ & \\
\hline Number of patients & 817 & 816 & 817 & \\
\hline Age, years & $67.6 \pm 15.7$ & $68.0 \pm 16.1$ & $68.9 \pm 15.2$ & 0.247 \\
\hline $\begin{array}{c}\text { Sex, n (\%) } \\
\text { Female } \\
\text { Male }\end{array}$ & $\begin{array}{l}440(53.9) \\
377(46.1)\end{array}$ & $\begin{array}{l}375(46.0) \\
44 I(54.0)\end{array}$ & $\begin{array}{l}354(43.3) \\
463(56.7)\end{array}$ & $<0.001$ \\
\hline $\begin{array}{l}\text { Ethnicity, n (\%) } \\
\text { Black } \\
\text { White } \\
\text { Other }\end{array}$ & $\begin{array}{l}578(70.7) \\
100(12.2) \\
139(17.0)\end{array}$ & $\begin{array}{c}630(77.2) \\
49(6.0) \\
137(16.8)\end{array}$ & $\begin{array}{c}632(77.4) \\
40(4.9) \\
145(17.7)\end{array}$ & $<0.001$ \\
\hline $\begin{array}{l}\text { Vital signs } \\
\text { Heart rate, beats/minute } \\
\mathrm{SBP}, \mathrm{mmHg} \\
\mathrm{DBP}, \mathrm{mmHg} \\
\mathrm{MAP}, \mathrm{mmHg} \\
\text { Respiratory rate, times/minute } \\
\text { Temperature, }{ }^{\circ} \mathrm{C} \\
\mathrm{SpO} 2, \%\end{array}$ & $\begin{array}{c}78.4 \pm 15.2 \\
129.9 \pm 17.6 \\
63.3 \pm 10.6 \\
83.1 \pm 11.1 \\
18.0 \pm 3.3 \\
36.8 \pm 0.6 \\
97.6 \pm 1.8\end{array}$ & $\begin{array}{c}79.1 \pm 14.5 \\
130.5 \pm 17.6 \\
64.1 \pm 11.7 \\
83.6 \pm 11.7 \\
18.2 \pm 3.6 \\
36.9 \pm 0.6 \\
97.2 \pm 3.1\end{array}$ & $\begin{array}{c}84.2 \pm 16.1 \\
127.2 \pm 18.3 \\
62.8 \pm 11.3 \\
81.7 \pm 11.9 \\
19.4 \pm 4.1 \\
37.0 \pm 0.7 \\
97.4 \pm 2.6\end{array}$ & $\begin{array}{l}<0.001 \\
<0.001 \\
<0.001 \\
<0.001 \\
<0.001 \\
<0.001 \\
0.140\end{array}$ \\
\hline $\begin{array}{l}\text { Laboratory parameters } \\
\text { SIRI, } 10^{9} / \mathrm{L} \\
\text { Neutrophil, \% } \\
\text { Monocyte, \% } \\
\text { Lymphocyte, \% } \\
\text { White blood cell counts, } 10^{9} / \mathrm{L} \\
\text { Platelet counts, } 10^{9} / \mathrm{L} \\
\text { Glucose, } \mathrm{mg} / \mathrm{dL} \\
\text { Blood urea nitrogen, } \mathrm{mg} / \mathrm{dl} \\
\text { Anion gap, } \mathrm{mg} / \mathrm{dl}\end{array}$ & $\begin{array}{c}0.9 \pm 0.4 \\
67.9 \pm 15.9 \\
4.0 \pm 2.3 \\
23.8 \pm 12.2 \\
8.9 \pm 11.1 \\
226.5 \pm 103.7 \\
138.7 \pm 57.1 \\
22.1 \pm 15.9 \\
15.0 \pm 3.4\end{array}$ & $\begin{array}{c}2.6 \pm 0.6 \\
81.6 \pm 8.0 \\
3.9 \pm 2.0 \\
12.5 \pm 4.9 \\
11.0 \pm 3.4 \\
243.9 \pm 95.6 \\
151.5 \pm 65.6 \\
22.5 \pm 16.0 \\
15.3 \pm 3.3\end{array}$ & $\begin{array}{c}10.9 \pm 14.2 \\
85.9 \pm 8.4 \\
4.6 \pm 3.6 \\
7.1 \pm 3.4 \\
16.5 \pm 13.9 \\
260.8 \pm 123.1 \\
163.8 \pm 68.3 \\
27.3 \pm 21.9 \\
16.3 \pm 3.9\end{array}$ & $\begin{array}{l}<0.001 \\
<0.001 \\
<0.001 \\
<0.001 \\
<0.001 \\
<0.001 \\
0.001 \\
<0.001 \\
<0.001\end{array}$ \\
\hline $\begin{array}{l}\text { Comorbidities, } \mathbf{n} \text { (\%) } \\
\text { Atrial fibrillation } \\
\text { CAD } \\
\text { Liver disease }{ }^{\$} \\
\text { Respiratory failure } \\
\text { Pneumonia }\end{array}$ & $\begin{array}{c}208(25.5) \\
148(18.1) \\
21(2.6) \\
168(20.6) \\
123(15.1)\end{array}$ & $\begin{array}{c}239(29.3) \\
147(18.0) \\
16(2.0) \\
219(26.8) \\
177(21.7)\end{array}$ & $\begin{array}{c}251(30.7) \\
149(18.2) \\
26(3.2) \\
297(36.4) \\
253(31.0)\end{array}$ & $\begin{array}{c}0.051 \\
0.993 \\
0.296 \\
<0.001 \\
<0.001\end{array}$ \\
\hline $\begin{array}{l}\text { Scoring systems } \\
\text { SOFA } \\
\text { SAPSII } \\
\text { GCS } \\
\text { APSIII }\end{array}$ & $\begin{array}{c}3.4 \pm 2.7 \\
34.8 \pm 13.3 \\
12.9 \pm 3.2 \\
40.8 \pm 19.7\end{array}$ & $\begin{array}{c}3.5 \pm 2.6 \\
34.9 \pm 13.0 \\
12.7 \pm 3.3 \\
40.2 \pm 19.2\end{array}$ & $\begin{array}{c}4.4 \pm 3.1 \\
41.0 \pm 14.2 \\
12.3 \pm 3.8 \\
47.8 \pm 22.6\end{array}$ & $\begin{array}{l}<0.001 \\
<0.001 \\
0.003\end{array}$ \\
\hline $\begin{array}{l}\text { Clinical outcomes, n (\%) } \\
\text { 30-day mortality } \\
\text { 90-day mortality } \\
\text { One-year mortality } \\
\text { In-hospital mortality } \\
\text { Sepsis }\end{array}$ & $\begin{array}{l}141(17.3) \\
173(21.2) \\
219(26.8) \\
163(20.0) \\
193(23.6)\end{array}$ & $\begin{array}{l}161(19.7) \\
201(24.6) \\
242(29.7) \\
190(23.3) \\
232(28.4)\end{array}$ & $\begin{array}{l}235(28.8) \\
285(34.9) \\
332(40.6) \\
27 \mid(33.2) \\
330(40.4)\end{array}$ & $\begin{array}{l}<0.001 \\
<0.001 \\
<0.001 \\
<0.001 \\
<0.001\end{array}$ \\
\hline
\end{tabular}

Notes: $\mathrm{SIRI}$ is calculated using the counts of peripheral venous blood neutrophils $(N)$, monocytes $(M)$, and lymphocytes $(L)$ as follows: $S I R I=N * M / L ; D a t a$ were presented as the mean \pm SD and $n$.

Abbreviations: SIRI, systemic inflammation response index; SBP, systolic blood pressure; DBP, diastolic blood pressure; MAP, mean arterial pressure; SpO2, pulse oximetryderived oxygen saturation; CAD, coronary artery disease; SOFA, sequential organ failure assessment; SAPS II, simplified acute physiology score II; GCS, Glasgow Coma Scale; APSIII, Antiphospholipid syndrome. 

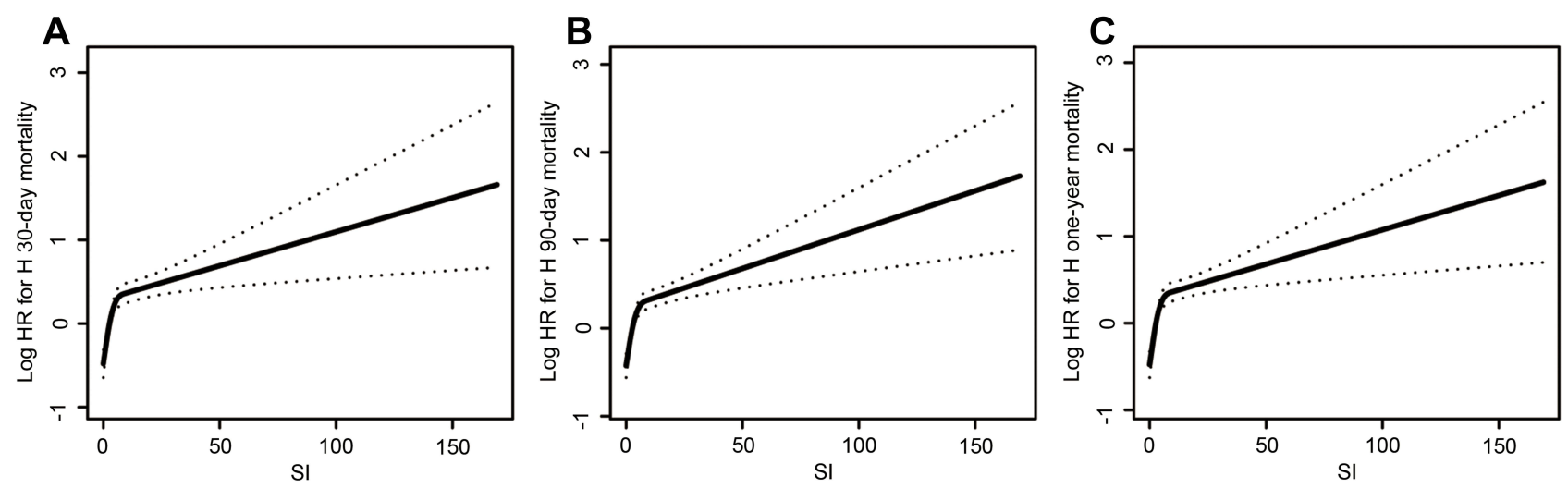

Figure I The relationship between systemic inflammatory response index (SIRI) and all-cause mortality. (A) 30-day mortality. (B) 90-day mortality. (C) one year mortality.

$0.5349 ; 0.6216$ vs $0.5628 ; 0.6216$ vs $0.5579 ; 0.6216$ vs

0.5865 , respectively).

\section{Association Between SIRI and NIHSS}

The results of the Pearson correlation analysis in Figure 3 suggested that SIRI was significantly and positively correlated with NIHSS; the correlation coefficient was 0.3404 $(p<0.001)$.

\section{Discussion}

The results above indicate that high SIRI values were associated with all-cause mortality in stroke patients after adjusting for several confounding factors. As evidenced by the smooth curve fitting results. High SIRI values were also accompanied by significantly higher in-hospital mortality, 30-day, 90-day and one-year mortality in stroke patients. Considering that potential confounders, including comorbidities and clinical parameters, may also affect the main outcome, the subgroup analysis showed that our results were reliable. Simultaneously we observed that SIRI was strongly correlated with the occurrence of sepsis; the higher the SIRI, the more likely it was to occur. It is widely acknowledged that the higher the NIHSS score, the more severe the stroke. In our study, SIRI was positively correlated with NIHSS; it can be concluded that SIRI and stroke severity are positively correlated.

A growing number of studies have demonstrated the relevance of inflammation in the pathogenesis of stroke. $^{22,23}$ Immune processes are usually activated by the ischemic cascade within minutes after stroke. Endovascular injury can trigger vascular occlusion and the chemotaxis of inflammatory cells into the brain parenchyma leading to tissue damage. Thus, inflammation plays a crucial role in stroke. ${ }^{24}$ Inflammation in ischemic stroke involves releasing cytokines, chemokines, and damage-associated molecular patterns that accentuate tissue destruction in the acute and repair stages of ischemic stroke. In addition, proinflammatory signals from immune mediators promptly activate permanent cells and affect the infiltration of various inflammatory cells (neutrophils, monocytes/macrophages, different subtypes of $\mathrm{T}$ cells and other inflammatory cells) into the ischemic zone intensifying the brain injury. ${ }^{25}$ The above mechanisms explain why currently developed biological markers are based on various inflammatory parameters associated with stroke, such as neutrophil-lymphocyte ratio (NLR) and platelet-lymphocyte ratio (PLR). ${ }^{26}$ Studies have shown a positive correlation between NLR and the risk of death at three months in stroke patients; ${ }^{27}$ an increase in PLR was predictive of the occurrence of post-stroke depression; ${ }^{28}$ a low LMR was independently related to a higher risk of hemorrhagic transformation in stroke patients ${ }^{29}$ and higher RDW could independently predict adverse outcomes in stroke patients. ${ }^{30}$ Due to the simple structure, single indicators of inflammation are not sufficient to present the severity of inflammation. Consequently, new biomarkers have been designed by combining different subtypes of white blood cells. $^{31,32}$

In contrast, as a novel chronic low-grade inflammatory indicator, SIRI consists of more comprehensive, easily accessible and cheap parameters. SIRI has received much attention recently as its significant role in predicting patient outcomes has been documented in diseases such as cancer, ${ }^{33}$ infectious diseases, ${ }^{34}$ and cardiovascular disease. ${ }^{35}$ However, to the best of our knowledge, no study has assessed the role of SIRI in predicting post-stroke mortality.

Studies have demonstrated that neutrophils are one of the first innate immune cells to respond to cerebral ischemia. ${ }^{4}$ Interestingly, neutrophils can aggravate inflammation in the 
Table 2 Association Between SIRI and Clinical Outcomes of in Critically III Patients with Stroke

\begin{tabular}{|c|c|c|c|c|c|c|}
\hline \multirow[t]{2}{*}{ Clinical Outcomes } & \multicolumn{2}{|c|}{ Model I } & \multicolumn{2}{|c|}{ Model 2} & \multicolumn{2}{|c|}{ Model 3} \\
\hline & HR (95\% Cl) & $P$ value & HR (95\% CI) & $P$ value & HR (95\% Cl) & $P$ value \\
\hline \multicolumn{7}{|l|}{ Primary outcome } \\
\hline \multicolumn{7}{|l|}{90 -day mortality ${ }^{\text {a }}$} \\
\hline Continuous variable & $1.01(1.01,1.02)$ & $<0.000$ I & $1.01(1.01,1.02)$ & $<0.0001$ & $1.01(1.01,1.02)$ & $<0.0033$ \\
\hline \multicolumn{7}{|l|}{ Tertile } \\
\hline$<1.6$ & 1.0 & & 1.0 & & 1.0 & \\
\hline $1.6-3.8$ & I.2I $(0.99,1.48)$ & 0.0684 & $1.19(0.97,1.46)$ & 0.0948 & $1.31(1.04,1.65)$ & 00.0967 \\
\hline$>3.8$ & $1.80(1.49,2.18)$ & $<0.0001$ & $1.75(1.45,2.1 \mathrm{I})$ & $<0.0001$ & $1.42(1.13,1.78)$ & 0.0128 \\
\hline$P$ for trend & & $<0.0001$ & & $<0.0001$ & & 0.0238 \\
\hline \multicolumn{7}{|l|}{ Secondary outcomes } \\
\hline \multicolumn{7}{|l|}{ 30-day mortality } \\
\hline Continuous variable & $1.01(1.01,1.02)$ & $<0.0001$ & $1.01(1.01,1.02)$ & $<0.0001$ & $1.01(1.01,1.02)$ & $<0.0033$ \\
\hline \multicolumn{7}{|l|}{ Tertile } \\
\hline$<1.6$ & 1.0 & & 1.0 & & 1.0 & \\
\hline $1.6-3.8$ & $1.19(0.95,1.49)$ & 0.1368 & $1.17(0.93,1.47)$ & 0.1721 & $1.16(0.92,1.47)$ & 0.2162 \\
\hline$>3.8$ & $1.79(1.46,2.21)$ & $<0.0001$ & $1.73(1.41,2.14)$ & $<0.0001$ & $1.26(1.00,1.59)$ & 0.0361 \\
\hline$P$ for trend & & $<0.0001$ & & $<0.0001$ & & 0.0461 \\
\hline \multicolumn{7}{|l|}{ One-year mortality ${ }^{a}$} \\
\hline Continuous variable & $1.01(1.01,1.02)$ & $<0.0001$ & $1.01(1.01,1.02)$ & $<0.0001$ & $1.01(1.00,1.02)$ & 0.0007 \\
\hline \multicolumn{7}{|l|}{ Tertile } \\
\hline$<1.6$ & 1.0 & & 1.0 & & 1.0 & \\
\hline $1.6-3.8$ & $1.15(0.96,1.38)$ & 0.1345 & $1.13(0.94,1.36)$ & 0.0387 & $1.13(0.93,1.37)$ & 0.2065 \\
\hline$>3.8$ & $1.69(1.43,2.01)$ & $<0.0001$ & $1.65(1.39,1.96)$ & $<0.0001$ & $1.28(1.06,1.54)$ & 0.0108 \\
\hline$P$ for trend & & $<0.0001$ & & $<0.0001$ & & 0.0128 \\
\hline \multicolumn{7}{|l|}{ In-hospital mortality } \\
\hline Continuous variable & $1.01(1.01,1.02)$ & $<0.0001$ & $1.01(1.01,1.02)$ & $<0.0001$ & $1.01(1.00,1.02)$ & 0.0039 \\
\hline \multicolumn{7}{|l|}{ Tertile } \\
\hline$<1.6$ & 1 & & I & & I & \\
\hline $1.6-3.8$ & I.2I $(0.98$, I.50) & 0.0700 & $1.20(0.97,1.48)$ & 0.0901 & $1.13(0.90,1.41)$ & 0.0901 \\
\hline$>3.8$ & $1.86(1.53,2.26)$ & $<0.0001$ & $1.82(1.50,2.21)$ & $<0.0001$ & $1.26(1.02,1.57)$ & 0.0330 \\
\hline$P$ for trend & & $<0.0001$ & & $<0.0001$ & & 0.0379 \\
\hline
\end{tabular}

Notes: ${ }^{a}$ Cox proportional hazards regression models were used to calculate hazard ratios (HR) with $95 \%$ confidence intervals (Cl). Model I covariates were adjusted for nothing. Model 2 covariates were adjusted for age, sex and ethnicity. Model 3 covariates were adjusted for age, sex, ethnicity, systolic blood pressure, diastolic blood pressure, heart rate, glucose, anion gap, temperature, platelet counts, atrial fibrillation, liver disease, respiratory failure, serum creatinine, hemoglobin GCS, SOFA.

Table 3 Association Between SIRI and Clinical Outcomes of Sepsis

\begin{tabular}{|c|c|c|c|c|c|c|}
\hline \multirow[t]{2}{*}{ Clinical Outcomes } & \multicolumn{2}{|c|}{ Model I } & \multicolumn{2}{|c|}{ Model 2} & \multicolumn{2}{|c|}{ Model 3} \\
\hline & OR (95\% Cl) & $P$ value & OR (95\% Cl) & $P$ value & OR (95\% CI) & $P$ value \\
\hline Continuous variable & $1.04(1.02,1.05)$ & $<0.0001$ & $1.04(1.02,1.05)$ & $<0.0001$ & $1.02(1.00,1.03)$ & 0.0085 \\
\hline Tertile & & & & & & \\
\hline$<1.6$ & 1.0 & & 1.0 & & 1.0 & \\
\hline $1.6-3.8$ & $1.28(1.03, I .60)$ & 0.0270 & $1.32(1.06,1.65)$ & 0.0145 & $1.20(0.92,1.57)$ & 0.1822 \\
\hline$>3.8$ & $2.19(1.77,2.71)$ & $<0.0001$ & $2.26(I .82,2.8 I)$ & $<0.0001$ & $1.45(1.10,1.91)$ & 0.0077 \\
\hline$P$ for trend & & $<0.0001$ & & $<0.0001$ & & 0.0095 \\
\hline
\end{tabular}

Notes: Logistic regression models were used to calculate odds ratios (OR) with $95 \%$ confidence intervals (Cl). Model I covariates were adjusted for nothing. Model 2 covariates were adjusted for age, sex and ethnicity. Model 3 covariates were adjusted for systolic blood pressure, diastolic blood pressure, system inflammatory response syndrome, serum creatinine, hemoglobin, white blood cell count, platelet count, red cell volume distribution width, atrial fibrillation, coronary artery disease, chronic kidney disease, respiratory failure, pneumonia, GCS and SOFA. 
Table 4 Subgroup Analysis of the Associations Between 90-Day All-Cause Mortality and the SIRI ${ }^{\mathrm{a}}$

\begin{tabular}{|c|c|c|c|}
\hline & No. of Patients & HR $(95 \% \mathrm{Cl})^{b}$ & $P$ for Interaction \\
\hline Age, years & & & 0.2759 \\
\hline$<70$ & 1225 & $1.02(1.01,1.03)$ & \\
\hline$\geq 71$ & 1225 & $1.01(1.00,1.02)$ & \\
\hline Gender & & & 0.8323 \\
\hline Male & $128 \mid$ & $1.01(1.01,1.02)$ & \\
\hline Female & 1169 & $\mathrm{I} .01(\mathrm{I} .01,1.02)$ & \\
\hline Ethnicity & & & 0.7819 \\
\hline White & 1840 & $1.01(1.01,1.02)$ & \\
\hline Black & 189 & $1.00(0.95,1.06)$ & \\
\hline Other & 421 & $1.02(1.00,1.03)$ & \\
\hline MAP, $\mathrm{mmHg}$ & & & 0.4585 \\
\hline$<82$ & 1222 & $1.01(1.01,1.02)$ & \\
\hline$\geq 83$ & 1222 & $1.02(1.01,1.04)$ & \\
\hline Heart rate, beats/minute & & & 0.8849 \\
\hline$<78$ & 1222 & $1.02(1.01,1.02)$ & \\
\hline$\geq 79$ & 1223 & $1.01(1.00,1.02)$ & \\
\hline Respiratory rate, times/minute & & & 0.8322 \\
\hline$<18$ & 1221 & $1.02(1.01,1.02)$ & \\
\hline$\geq 18$ & 1222 & $1.01(1.00,1.02)$ & \\
\hline Congestive hearts failure & & & 0.2162 \\
\hline Yes & 232 & I.0I $(0.98,1.04)$ & \\
\hline No & 2218 & $1.01(1.01,1.02)$ & \\
\hline Atrial fibrillation & & & 0.2627 \\
\hline Yes & 698 & $1.01(1.00,1.02)$ & \\
\hline No & 1752 & $1.02(1.01,1.02)$ & \\
\hline Liver disease & & & $0.774 I$ \\
\hline Yes & 63 & $1.09(1.05,1.13)$ & \\
\hline No & 2387 & $1.01(1.01,1.02)$ & \\
\hline Coronary artery disease & & & 0.4418 \\
\hline Yes & 444 & $1.01(1.00,1.02)$ & \\
\hline No & 2006 & $1.02(1.01,1.02)$ & \\
\hline Pneumonia & & & 0.1167 \\
\hline Yes & 553 & $1.02(1.01,1.03)$ & \\
\hline No & 1897 & $1.01(1.01,1.02)$ & \\
\hline Anion gap, mmol/l & & & 0.0494 \\
\hline$\leq 14$ & 994 & $1.01(1.01,1.02)$ & \\
\hline$\geq 15$ & 1447 & $1.02(1.00,1.03)$ & \\
\hline Glucose, mg/dl & & & 0.0952 \\
\hline$\leq 133$ & 1207 & $1.03(1.02,1.04)$ & \\
\hline$\geq 134$ & $|24|$ & $1.01(1.00,1.02)$ & \\
\hline Serum urea nitrogen, $\mathrm{mg} / \mathrm{dl}$ & & & 0.1809 \\
\hline$\leq 18$ & 1196 & $1.02(1.01,1.03)$ & \\
\hline$\geq 19$ & 1253 & $1.01(1.00,1.02)$ & \\
\hline
\end{tabular}


Table 4 (Continued).

\begin{tabular}{|c|c|c|c|}
\hline & No. of Patients & HR $(95 \% \mathrm{Cl})^{b}$ & $P$ for Interaction \\
\hline Potassium, mmol/l & & & 0.8999 \\
\hline$\leq 3.9$ & 1096 & 1.02 (I.01, I.02) & \\
\hline$\geq 4.0$ & 1354 & 1.01 (1.01, I.02) & \\
\hline Sodium, $\mathrm{mmol} / \mathrm{l}$ & & & 0.4633 \\
\hline$\geq 138$ & 1011 & 1.01 (I.01, I.02) & \\
\hline$\geq 139$ & 1438 & 1.02 (I.0I, I.02) & \\
\hline APTT, second & & & 0.8038 \\
\hline$\leq 26.6$ & 1193 & $1.02(1.01,1.03)$ & \\
\hline$>26.7$ & 1217 & 1.01 (I.0I, I.02) & \\
\hline INR & & & 0.0214 \\
\hline$\leq 1.0$ & 578 & $1.02(0.99,1.05)$ & \\
\hline$\geq 1.1$ & 1836 & 1.01 (I.0I, I.02) & \\
\hline PT, second & & & 0.6098 \\
\hline$\leq 13.1$ & 1140 & $1.02(1.00,1.04)$ & \\
\hline$\geq 13.2$ & 1274 & I.0I (I.0I, I.02) & \\
\hline SAPSII & & & 0.3413 \\
\hline$\leq 34$ & 1154 & $1.05(1.02,1.08)$ & \\
\hline$\geq 35$ & 1296 & $1.01(1.00,1.01)$ & \\
\hline APSIII & & & 0.1427 \\
\hline$\leq 37$ & 1189 & $1.03(1.02,1.05)$ & \\
\hline$\geq 38$ & $|26|$ & $1.01(1.00,1.01)$ & \\
\hline
\end{tabular}

Notes: Cox proportional hazards regression models were used to calculate hazard ratios (HR) with $95 \%$ confidence intervals $(\mathrm{Cl})$. ${ }^{\mathrm{a}} \mathrm{Cox}$ proportional hazards regression models were used to calculate hazard ratios (HR) with $95 \%$ confidence intervals $(\mathrm{Cl})$. ${ }^{\mathrm{b}}$ covariates were adjusted for age, sex and ethnicity.

brain parenchyma by liberating multiple pro-inflammatory mediators. The homeostasis of its damage is connected with



Figure 2 Receiver operating curve (ROC) for prediction in stroke patients using systemic inflammatory response index (SIRI). (AUC: SIRI: 0.622; RDW: 0.587; PLR:0.563; LMR: 0.558; NLR:0.535, separately). stroke severity by influencing systemic inflammation and the blood-brain barrier (BBB) ${ }^{36}$ Inflammatory reactions can cause secondary tissue injury in the brain, ${ }^{37}$ and the adhesion of neutrophils to endothelial cells has been documented as the basis of inflammation. ${ }^{38}$ Besides, other immune cells have been reported to play a significant role in ischemic stroke. For instance, cerebral ischemia and hypoxia can stimulate monocytes to generate inflammatory mediators, such as interleukins-6 (IL-6) and tumor necrosis factor (TNF), further worsening cerebral ischemia and hypoxia, leading to more extensive brain tissue destruction. ${ }^{39}$ Alternatively, monocytes can activate platelets to become platelet-monocyte aggregates (PMA), facilitating the liberation of an inflammatory response, adhesion, and vasoactive substances. The PMA can also promote thrombosis and vascular occlusion, causing hemodynamic changes and exacerbating the cerebral ischemic injury. ${ }^{40}$ Another study suggested that microglia exhaustion could intensify neuroinflammation in the brain after ischemia. Studies showed that microglia depletion enhanced leukocyte infiltration, neuronal death and inflammatory mediator release as well as enlarged 
Table 5 Receiver Operating Curve (ROC) for Prediction in Stroke Patients

\begin{tabular}{|l|c|c|c|}
\hline & ROC Area (AUC) & 95\% CI Low & 95\% CI Upp \\
\hline NLR & 0.5349 & 0.5102 & 0.5598 \\
PLR & 0.5628 & 0.5378 & 0.5879 \\
LMR & 0.5579 & 0.5332 & 0.5825 \\
RDW & 0.5865 & 0.5618 & 0.6113 \\
SIRI & 0.6216 & 0.5979 & 0.6453 \\
\hline
\end{tabular}

Abbreviations: AUC, area under the curve; $\mathrm{Cl}$, confidence interval; NLR, neutrophil-to-lymphocyte ratio; PLR, platelet-to-lymphocyte ratio; LMR, lymphocyte to monocyte ratio; RDW, red blood cell distribution width; SIRI, systemic inflammation response index.

brain infarct size in stroke patients. ${ }^{41}$ Microglia are more likely to release cytotoxic factors in a severe ischemic environment compared to a mild ischemic environment. ${ }^{42}$ Apart from it, lymphocytes can coordinate the inflammatory response. However, explaining the role of lymphocytes in stroke is complicated due to the huge diversity of lymphocytes. ${ }^{43} \mathrm{~T}$ lymphocytes have been found to play both beneficial and detrimental roles in stroke. Natural killer (NK) cells exacerbate brain damage by catalyzing neuronal death. ${ }^{44}$ In contrast, T regulatory cells (Tregs) are generally involved in suppressing inflammation and regulating and maintaining homeostasis and immune tolerance in the periphery. Furthermore, Tregs secreting the cytokine IL-10 demonstrate protection from stroke. Studies indicated that animals with increased numbers of post-stroke Tregs exhibited better outcomes post-stroke. ${ }^{45}$

In the present study, we found that SIRI was positively correlated with NIHSS. Since NIHSS is commonly used in clinical practice to assess the severity of a stroke, we can conclude that SIRI was closely related to stroke severity. To the best of our knowledge, no studies have previously

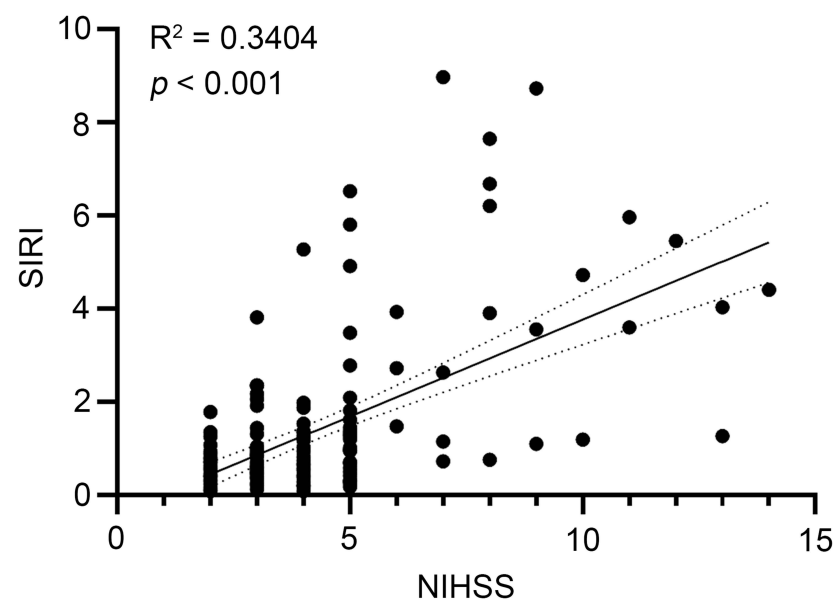

Figure 3 Pearson's correlation test to analyze the relationship between systemic inflammatory response index (SIRI) and National Institute of Health stroke scale (NIHSS). $\left(R^{2}=0.3404, p<0.001\right)$. documented the role of SIRI in stroke prognosis. Accordingly, there is great potential to use SIRI as a predictor of stroke prognosis.

There were many limitations in this study. First, subjects in this study were from only one region, which may have caused selection bias or geographically biased results; the next step will be conducting a multi-center study. Moreover, the number of covariates related to stroke prognosis was extremely large and was inadequately collected in our study. More data on other parameters is essential to improve the robustness of our results. Finally, the sample size of our study was relatively small; greater sample sizes are necessary to substantiate our findings.

\section{Conclusions}

We delivered the first study demonstrating that SIRI is strongly correlated with stroke mortality and severity and the risk of sepsis. The higher the SIRI value, the worse the stroke prognosis. Furthermore, SIRI exhibited better ability in predicting stroke prognosis than NLR, PLR, LMR and RDW. Accordingly, SIRI may be a new promising low-grade inflammatory indicator for predicting the prognosis of stroke.

\section{Acknowledgments}

Thanks to the team of Dr. Hanmin Wang of the Department of Neurology at the First Hospital of Wenzhou Medical University for the help and support.

\section{Disclosure}

The authors report no conflicts of interest in this work.

\section{References}

1. Knight-Greenfield A, Nario JJQ, Gupta A. Causes of acute stroke: a patterned approach. Radiol Clin North Am. 2019;57(6):1093-1108. doi:10.1016/j.rcl.2019.07.007

2. Wu S, Wu B, Liu M, et al.; China Stroke Study Collaboration. Stroke in China: advances and challenges in epidemiology, prevention, and management. Lancet Neurol. 2019;18(4):394-405. doi:10.1016/ S1474-4422(18)30500-3. 
3. Hathidara MY, Saini V, Malik AM. Stroke in the young: a global update. Curr Neurol Neurosci Rep. 2019;19(11):91. doi:10.1007/ s11910-019-1004-1

4. Dong X, Gao J, Su Y, Wang Z. Nanomedicine for ischemic stroke. Int J Mol Sci. 2020;21(20):7600. doi:10.3390/ijms21207600

5. Maida CD, Norrito RL, Daidone M, Tuttolomondo A, Pinto A. Neuroinflammatory mechanisms in ischemic stroke: focus on cardioembolic stroke, background, and therapeutic approaches. Int $J \mathrm{Mol}$ Sci. 2020;21(18):6454. doi:10.3390/ijms21186454

6. Chauhan G, Debette S. Genetic risk factors for Ischemic and hemorrhagic stroke. Curr Cardiol Rep. 2016;18(12):124. doi:10.1007/ s11886-016-0804-z

7. Anrather J, Iadecola C. Inflammation and stroke: an overview. Neurotherapeutics. 2016;13(4):661-670. doi:10.1007/s13311-0160483-x

8. Shi K, Tian DC, Li ZG, Ducruet AF, Lawton MT, Shi FD. Global brain inflammation in stroke. Lancet Neurol. 2019;18 (11):1058-1066. doi:10.1016/S1474-4422(19)30078-X

9. Lambertsen KL, Finsen B, Clausen BH. Post-stroke inflammation-target or tool for therapy? Acta Neuropathol. 2019;137(5):693-714. doi:10.1007/s00401-018-1930-z

10. Shekhar S, Cunningham MW, Pabbidi MR, Wang S, Booz GW, Fan F. Targeting vascular inflammation in ischemic stroke: recent developments on novel immunomodulatory approaches. Eur J Pharmacol. 2018;833:531-544. doi:10.1016/j.ejphar.2018.06.028

11. He Q, Li L, Ren Q. The prognostic value of preoperative Systemic Inflammatory Response Index (SIRI) in patients with high-grade glioma and the establishment of a nomogram. Front Oncol. 2021;11:671811. doi:10.3389/fonc.2021.671811

12. Chen L, Kong X, Wang Z, Wang X, Fang Y, Wang J. Pretreatment systemic inflammation response index in patients with breast cancer treated with neoadjuvant chemotherapy as a useful prognostic indicator. Cancer Manag Res. 2020;12:1543-1567. doi:10.2147/ CMAR.S235519

13. He WZ, Jiang C, Liu LL. etc. Association of body composition with survival and inflammatory responses in patients with non-metastatic nasopharyngeal cancer. Oral Oncol. 2020;108:104771. doi:10.1016/j. oraloncology.2020.104771

14. Jia CP, Chen H, Sun B. Research advances on the value of preoperative systemic inflammatory response index in predicting the prognosis of patients with resectable pancreatic cancer. Zhonghua Wai Ke Za Zhi. 2019;57(11):862-865. Chinese. doi:10.3760/cma.j.issn.05295815.2019.11.013

15. Wanrooy BJ, Wen SW, Wong CH. Dynamic roles of neutrophils in post-stroke neuroinflammation. Immunol Cell Biol. 2021;99 (9):924-935. doi:10.1111/imcb.12463

16. Cai W, Liu S, Hu M, et al. Functional dynamics of neutrophils after ischemic stroke. Transl Stroke Res. 2020;11(1):108-121. doi:10.1007/s12975-019-00694-y

17. Han D, Liu H, Gao Y. The role of peripheral monocytes and macrophages in ischemic stroke. Neurol Sci. 2020;41(12):3589-3607. doi:10.1007/s10072-020-04777-9

18. Meng H, Zhao H, Cao X, et al. Double-negative T cells remarkably promote neuroinflammation after ischemic stroke. Proc Natl Acad Sci USA. 2019;116(12):5558-5563. doi:10.1073/pnas.1814394116

19. Doyle KP, Buckwalter MS. Does B lymphocyte-mediated autoimmunity contribute to post-stroke dementia? Brain Behav Immun. 2017;64:1-8. doi:10.1016/j.bbi.2016.08.009

20. Jin Z, Hao D, Song Y, Zhuang L, Wang Q, Yu X. Systemic inflammatory response index as an independent risk factor for ischemic stroke in patients with rheumatoid arthritis: a retrospective study based on propensity score matching. Clin Rheumatol. 2021;40 (10):3919-3927. doi:10.1007/s10067-021-05762-z

21. Johnson AEW, Pollard TJ, Shen L, et al. MIMIC-III, a freely accessible critical care database. Sci Data. 2016;3(1):160035. doi:10.1038/ sdata.2016.35
22. Khoshnam SE, Winlow W, Farzaneh M, Farbood Y, Moghaddam HF. Pathogenic mechanisms following ischemic stroke. Neurol Sci. 2017;38(7):1167-1186. doi:10.1007/s10072-017-2938-1

23. Tuttolomondo A. Ischemic stroke pathogenesis: genetics, epigenetics and inflammation. Curr Pharm Des. 2020;26(34):4207-4208. doi: $10.2174 / 138161282634200831110542$

24. Nakamura K, Shichita T. Cellular and molecular mechanisms of sterile inflammation in ischaemic stroke. $J$ Biochem. 2019;165 (6):459-464. doi:10.1093/jb/mvz017

25. Jayaraj RL, Azimullah S, Beiram R, Jalal FY, Rosenberg GA. Neuroinflammation: friend and foe for ischemic stroke. $J$ Neuroinflammation. 2019;16(1):142. doi:10.1186/s12974-019$1516-2$

26. Süße M, Hannich MJ, Holbe C, Ruhnau J, von Sarnowski B, Dressel A. Intrathecal inflammation in young stroke. Acta Neurol Scand. 2019;140(1):9-16. doi:10.1111/ane.13094

27. Nam KW, Kim TJ, Lee JS, et al. High neutrophil-to-lymphocyte ratio predicts stroke-associated pneumonia. Stroke. 2018;49(8):1886-1892. doi:10.1161/STROKEAHA.118.021228

28. Huang G, Chen H, Wang Q, et al. High platelet-to-lymphocyte ratio are associated with post-stroke depression. J Affect Disord. 2019;246 (246):105-111. doi:10.1016/j.jad.2018.12.012

29. Park J, Chang JY, Kim JY, Lee JE. Monocyte transmodulation: the next novel therapeutic approach in overcoming ischemic stroke? Front Neurol. 2020;11:578003. doi:10.3389/fneur.2020.578003

30. Feng GH, Li HP, Li QL, Fu Y, Huang RB. Red blood cell distribution width and ischaemic stroke. Stroke Vasc Neurol. 2017;2(3):172-175. doi:10.1136/svn-2017-000071

31. Hu J, Zhou W, Zhou Z, Han J, Dong W. Elevated neutrophil-tolymphocyte and platelet-to-lymphocyte ratios predict post-stroke depression with acute ischemic stroke. Exp Ther Med. 2020;19 (4):2497-2504. doi:10.3892/etm.2020.8514

32. Ma GG, Tu GW, Zheng JL, et al. Changes in stroke volume variation induced by passive leg raising to predict fluid responsiveness in cardiac surgical patients with protective ventilation. $J$ Cardiothorac Vasc Anesth. 2020;34(6):1526-1533. doi:10.1053/j.jvca.2019.10.002

33. Pacheco-Barcia V, Mondéjar SR, France T, et al. A systemic inflammation response index (SIRI) correlates with survival and predicts oncological outcome for mFOLFIRINOX therapy in metastatic pancreatic cancer. Pancreatology. 2020;20(2):254-264. doi:10.1016/j. pan.2019.12.010

34. Hui DS, Lee N, Chan PK, Beigel JH. The role of adjuvant immunomodulatory agents for treatment of severe influenza. Antiviral Res. 2018;150:202-216. doi:10.1016/j.antiviral.2018.01.002

35. Ferrara D, Montecucco F, Dallegri F, Carbone F. Impact of different ectopic fat depots on cardiovascular and metabolic diseases. J Cell Physiol. 2019;234(12):21630-21641. doi:10.1002/jcp.28821

36. Otxoa-de-amezaga A, Miró-Mur F, Pedragosa J, et al. Microglial cell loss after ischemic stroke favors brain neutrophil accumulation. Acta Neuropathol. 2019;137(2):321-341. doi:10.1007/s00401-018-1954-4

37. Low A, Mak E, Rowe JB, Markus HS, O'Brien JT. Inflammation and cerebral small vessel disease: a systematic review. Ageing Res Rev. 2019;53:100916. doi:10.1016/j.arr.2019.100916

38. Dong X, Gao J, Zhang CY, Hayworth C, Frank M, Wang Z. Neutrophil membrane-derived nanovesicles alleviate inflammation to protect mouse brain injury from ischemic stroke. ACS Nano. 2019;13(2):1272-1283. doi:10.1021/acsnano.8b06572

39. Kanazawa M, Ninomiya I, Hatakeyama M, Takahashi T, Shimohata T. Microglia and monocytes/macrophages polarization reveal novel therapeutic mechanism against stroke. Int J Mol Sci. 2017;18(10):2135. doi:10.3390/ijms18102135

40. Tabib A, Hindi I, Karbian N, Zelig O, Falach B, Mevorach D. Prothrombotic mechanisms in patients with congenital p.Cys89Tyr mutation in CD59. Thromb Res. 2018;168:67-77. doi:10.1016/j. thromres.2018.06.006 
41. Ma Y, Wang J, Wang Y, Yang GY. The biphasic function of microglia in ischemic stroke. Prog Neurobiol. 2017;157:247-272. doi:10.1016/ j.pneurobio.2016.01.005

42. Wang J, Xing H, Wan L, Jiang X, Wang C, Wu Y. Treatment targets for M2 microglia polarization in ischemic stroke. Biomed Pharmacother. 2018;105:518-525. doi:10.1016/j.biopha.2018.05.143

43. Stoll G, Nieswandt B. Thrombo-inflammation in acute ischaemic stroke - implications for treatment. Nat Rev Neurol. 2019;15 (8):473-481. doi:10.1038/s41582-019-0221-1
44. Liu Q, Jin WN, Liu Y. Brain ischemia suppresses immunity in the periphery and brain via different neurogenic innervations. Immunity. 2017;46(3):474-487. doi:10.1016/j.immuni.2017.02.015

45. Selvaraj UM, Stowe AM. Long-term T cell responses in the brain after an ischemic stroke. Discov Med. 2017;24(134):323-333.
Clinical Interventions in Aging

\section{Publish your work in this journal}

Clinical Interventions in Aging is an international, peer-reviewed journal focusing on evidence-based reports on the value or lack thereof of treatments intended to prevent or delay the onset of maladaptive correlates of aging in human beings. This journal is indexed on PubMed Central, MedLine, CAS, Scopus and the Elsevie
Dovepress

Bibliographic databases. The manuscript management system is completely online and includes a very quick and fair peer-review system, which is all easy to use. Visit http://www.dovepress.com/ testimonials.php to read real quotes from published authors.

Submit your manuscript here: https://www.dovepress.com/clinical-interventions-in-aging-journal 